\title{
Kolliphor® HS 15 Micelles for the Delivery of Coenzyme Q10: Preparation, Characterization, and Stability
}

\author{
Li Liu, ${ }^{1,2}$ Kai Mao, ${ }^{1,2}$ Wenting Wang, ${ }^{1,2}$ Hongchun Pan, ${ }^{1,2,5}$ Fen Wang, ${ }^{3}$ Min Yang, ${ }^{4}$ and Hong Liu ${ }^{1,2,5}$
}

Received 22 March 2015; accepted 19 August 2015; published online 4 September 2015

\begin{abstract}
To enhance the stability of coenzyme Q10 $\left(\mathrm{CoQ}_{10}\right)$, Kolliphor ${ }^{\circledR}$ HS 15 (HS15) was employed as a carrier to build up a stable CoQ10-loaded micelle delivery system. The impact of micellar compositions, the preparation condition, and the preparation method on size characteristics, the solubilization efficiency, and micellar stability were investigated. The optimal preparation conditions were 1:6, 4, $0.2 \%, 118^{\circ} \mathrm{C}$, and $25 \mathrm{~min}$ for $\mathrm{CoQ} 10 / \mathrm{HS} 15$ mass ratio, $\mathrm{pH}$ value, the concentration of glucose, and the sterilization conditions. Upon these conditions, the particle size, polydispersity index (PDI), zeta potential, the entrapment efficiency, drug loading, and the critical micelle concentration (CMC) of CoQ10-loaded micelles were $19.76 \mathrm{~nm}, 0.112,-3.405 \mathrm{mV}, 99.39 \%, 13.77 \%$, and $5.623 \times 10^{-4} \mathrm{~g} / \mathrm{mL}$, respectively. Differential scanning calorimetry (DSC) analysis collectively corroborated that CoQ10 was entrapped into the micelles in amorphous form. The release pattern of drug was analyzed and proved to follow the first order. Additionally, the samples were exposed to the temperatures of $30^{\circ} \mathrm{C}$ for 6 months with more significant impact on their stabilities as compared to 4 and $25^{\circ} \mathrm{C}$ based on particle size and PDI. Under constant humidity with light protection long-term $\left(25 \pm 2^{\circ} \mathrm{C}\right.$, relative humidity $(\mathrm{RH}) 60 \pm 10 \%, 18$ months) conditions, there was no variation except minor changes of CoQ10 content of the samples. The shelf life of the micellar samples could be predicted as 24 months based on the stability results. Consequently, the CoQ10loaded micelles showed excellent stabilities below $25^{\circ} \mathrm{C}$ as a potential drug candidate for further clinical applications.
\end{abstract}

KEY WORDS: coenzyme $\mathrm{Q}_{10}$; Kolliphor ${ }^{\circledR}$ HS 15; micelles; preparation; stability.

\section{INTRODUCTION}

Coenzyme Q10 (CoQ10) is a fat-soluble vitamin analog found in the cells of many organisms, especially the inner mitochondrial membrane (1). In eukaryote, it functions as a cofactor in the electron transport chain and serves as a modulator of the transition pore (2). Hence, CoQ10 is a wellknown antioxidant, which regenerates alpha-tocopherol, another critical antioxidant, scavenges free radicals, and inhibits lipid peroxidation (3). Nevertheless, the CoQ10 level is often found to be deficient in several disease forms (4). CoQ10 is now diffusely applied as a supplement in cardiovascular health (5).

\footnotetext{
${ }^{1}$ College of Pharmaceutical Sciences, Southwest University, 2 Tiansheng Road, Beibei, Chongqing, 400716, People's Republic of China.

${ }^{2}$ Chongqing Engineering Research Center for Pharmaceutical Process and Quality Control, Chongqing, People's Republic of China.

${ }^{3}$ Jiangsu Simcere Pharmaceutical R\&D Co.Ltd, Nanjing, People's Republic of China.

${ }^{4}$ Sichuan Kelun Pharmaceutical Research Institute Co., Ltd., Chengdu, People's Republic of China.

${ }^{5}$ To whom correspondence should be addressed. (e-mail: panhongchun216@126.com; lhphch@126.com)
}

In spite of its versatility, poor bioavailability is one of the limiting factors in the clinical development of CoQ10 (6) which is mainly caused by its extremely low solubility in water $(<0.25 \mu \mathrm{g} / \mathrm{ml})(7,8)$. Various efforts have been made for improving the solubility of CoQ10 using a capable vehicle to enclose hydrophobic, such as inclusion complexes with microemulsions, dendrimers, or protein formulation (7,9-11). The administration of many cosolvents or surfactants causes toxicity and other undesirable side effects (12). Another option is to use certain micelle-forming surfactants in formulations of insoluble drugs. Thus, the requirement of an ideal drug delivery system comprises efficient and stable solubilization, which enhances the solubility of hydrophobic solutes via micellar formation at the concentrations above the critical micelle concentration (CMC). As a drug vector, micellar carrier is one of the most important entities designed essentially for effective transportation of loaded drug to the target site, which sequesters transport and retains the active drug until its site-specific delivery (13). Polymeric micelles by amphiphilic diblock copolymers are generally more stable and safer than conventional surfactant ones. However, critical problems relating to toxicity, caused by residual monomers or oligomers, and the drug-to-monomer cross-reaction may limit potential use $(11,14)$. Besides, the complexity of preparing limits the development of clinical studies. The preparation of conventional surfactant micelles is simple and feasible, on 
the contrary. Hence, exploring an efficient and stable solubilizer is still desired.

Solubilizers currently used for parenteral administration such as Cremophor® EL and polysorbate 80 have been implicated in clinically important adverse effects such as hypersensitivity reactions and a highly increased systemic drug exposure along with a reduced cellular uptake (15). To overcome these problems, they are replaced with a new nonionic surfactant called Kollipher® HS 15 (HS15; polyglycol monoester and diester of 12-hydroxystearic acid with about $30 \%$ free polyethylene glycol) (16), which is known to be safe in various animal toxicity models with reduced histamine levels compared with Cremophor ${ }^{\circledR}$ EL and polysorbate 80 (17). HS15 is official in the German Pharmacopoeia (DAB), US Pharmacopoeia (USP), and European Pharmacopoeia ( $\mathrm{Ph}$. Eur) monographs, and it has an import registration certificate in China. HS15 has been found to enable poorly soluble drugs to enhance the solubilization (18-20) and to inhibit to some degree the P-glycoprotein efflux mechanism (21,22). Thus, HS15 greatly increases the transportation of drug in the systemic circulation for poorly soluble drugs, further boosting the bioavailability.

In this contribution, HS15 was applied to produce a novel CoQ10-loaded micellar system to enhance the physicochemical stability of CoQ10. CoQ10, a promising antioxidant, was controllably incorporated within the micelles, since the marketed formulation $\mathrm{Hefu}{ }^{\circledR}$ was easy to crystallize. The physical and chemical properties including major parameters as the particle size, the particle dispersion index (PDI), zeta potential, the content of CoQ10, and the related substance have been investigated to optimize the preparation conditions of CoQ10-loaded micellar system. The obtained CoQ10loaded micelles were evaluated by size characteristics, solubilization efficiency, and the micellar stability. Finally, the shelf life of 24 months was obtained, and the CoQ10-loaded micelle system has good stability under $25^{\circ} \mathrm{C}$.

\section{MATERIALS}

\section{Materials}

CoQ10 was purchased from Shanxi Sciphar Hi-Tech Industrial Co., Ltd. (Shanxi, China); HS15 was kindly supplied by BASF Co., Ltd. (Shanghai, China). Polysorbate 80 was obtained from Nanjing Well Chemical Co., Ltd. (Nanjing, China). Methanol and ethanol were purchased from Spectrum Chemical Mfg. Corp. (HPLC grade, Gardena, USP).

\section{Methods}

\section{Solubilization Capacity Determination}

Excess amounts of CoQ10 were respectively added to $10 \mathrm{~mL}$ of different micellar solutions (HS15 or polysorbate $80)$ in vials which were then shaken in a thermostatted water bath (DF-101S; Kefeng Equipment Co., Ltd., Zhengzhou, China) at $50^{\circ} \mathrm{C}$ for $24 \mathrm{~h}$. Then, excess amounts of the poorly soluble CoQ10 were separated by $10 \mathrm{~min}$ centrifugation at $10,000 \mathrm{rpm}$ in a centrifuge (H2050R-1; Xiangyi Centrifuge Machines Ltd., Hunan, China). CoQ10 concentration in the supernatant solutions was determined by using HPLC (23).
All data were performed as mean \pm standard deviation (SD), $n=3$.

\section{Determination of Hemolytic Activity of HS15}

The hemolytic activity of HS15 micelles was investigated by rabbit red blood cells (RBCs) with polysorbate 80 used as a control. Briefly, RBC suspension $(25 \mathrm{~mL})$ was added into $25 \mathrm{~mL}$ of samples to make the concentrations of HS15 and polysorbate 80 of 120,240 , and $600 \mu \mathrm{g} / \mathrm{mL}$. The samples were incubated at $37^{\circ} \mathrm{C}$ for $24 \mathrm{~h}$, and $6 \mathrm{~mL}$ of the solution was taken at a desired time interval and then centrifuged at $3000 \mathrm{rpm}$ for $10 \mathrm{~min}$ to remove intact RBCs. The supernatant was analyzed for released hemoglobin with a UV-1800 spectrophotometer (Mapuda, China) at $540 \mathrm{~nm}$. Normal saline and distilled water were added to obtain 0 and $100 \%$ hemolysis, respectively. The degree of hemolysis was calculated by the following equation:

Hemolysis $(\%)=\frac{A_{\mathrm{s}}-A_{\mathrm{o}}}{A_{100}-A_{0}} \times 100$

Specifically, $A_{\mathrm{s}}$ is the absorbance of a sample, and $A_{100}$ and $A_{0}$ are the absorbance of the reference solutions at $100 \%$ hemolysis and $0 \%$ hemolysis, respectively (24).

\section{Preparation of CoQ10-Loaded Micelles}

The preparation method consisted of the following steps. In brief, specified amounts of HS15 (from 10 to $40 \mathrm{mg}$ ) and CoQ10 (5 mg) in a glass beaker were dissolved in a thermostatted water bath at $50^{\circ} \mathrm{C}$ and stirred for $15 \mathrm{~min}$. Then, $3 \mathrm{~mL}$ of $0.1538 \mathrm{M}$ sodium chloride solution containing stabilizer was added drop by drop while stirring. Subsequent solution was introduced for diluting the micellar solution to $50 \mathrm{~mL}$. Thus, the $\mathrm{pH}$ of the solution was adjusted with $0.1 \mathrm{M} \mathrm{HCl}$ and $\mathrm{NaOH}$. The samples were filtered using a syringe filter. Eventually, the subjects were sterilized as shown in subsequent section.

In the preliminary experiments, such influence factors such as types and amount of solubilizer, temperature, hydration time, and volume were investigated to identify the control factors and their levels during assembly process with main particle size, PDI, entrapment efficiency $(\mathrm{EE} \%)$, and so on as index. According to the influence of each factor, the ratio $(w / w)$ of CoQ10 and HS15, the amount of stabilizer, $\mathrm{pH}$ value, and the condition of sterilization served as four key factors to obtain the optimized formulation.

Screening of HS15 Concentration. CoQ10 was dissolved in HS15, in ratios of $1: 2,1: 3,1: 4,1: 5,1: 6,1: 7$, and 1:8, respectively. The most suitable ratio was identified on the basis of transmittance, the particle size, PDI, entrapment efficiency, and drug loading capacity.

Screening of Suitable Stabilizer and Its Concentration. CoQ10loaded micelles were prepared by using different types and concentrations of stabilizers such as glucose, cysteine, thiourea, and sodium pyrosulfite. The ratio of CoQ10 to HS15 (1:6) was kept constant. The most suitable stabilizer and its concentration were 
identified based on the content of CoQ10 and the related substance.

Screening of $\mathrm{pH}$. Screening of $\mathrm{pH}$ aimed to get the optimum CoQ10-loaded micelles. Toward this purpose, micelles were prepared following the above described process with the ratio of CoQ10 to HS15 at 1:6 and the glucose concentration at $0.2 \%(w / v)$. Different $\mathrm{pH}$ values were adjusted for preparation of micelles, and optimum $\mathrm{pH}$ values were selected based on the particle size, PDI, zeta potential, content of CoQ10, and related substance.

Optimization of Sterilization. CoQ10-loaded micelles were prepared using different sterilization conditions, i.e., $25 \mathrm{~min}$ at $118^{\circ} \mathrm{C}$ and $20 \mathrm{~min}$ at $121^{\circ} \mathrm{C}$, and its effect on the particle size, PDI, zeta potential, content of CoQ10, and related substance were studied. Other experimental parameters, including micellar compositions (CoQ10/HS15 (w/w) 1:6), glucose concentration $(0.2 \% w / v)$, and $\mathrm{pH} 4.0$, were kept constant.

\section{Characterization of CoQ10-Loaded Micelles}

Particles Size and Zeta Potential Measurement. The micellar sizes, size distributions, and zeta potential were determined by dynamic light scattering (DLS) using a Zetasizer Nano (Malvern, UK). To be specific, samples were analyzed at $25^{\circ} \mathrm{C}$ at an angle of $90^{\circ}$.

Entrapment Efficiency and Drug Loading Capacity. Micellar entrapment efficiency (EE\%) and drug loading capacity (DL\%) were determined using the centrifugation technique (25) for separating the nonentrapped drug from micelles. The content of CoQ10 in CoQ10-loaded micelles (without centrifugation was $m_{1}$, with centrifugation was $m_{2}$ ) was determined by using a validated HPLC method (1260, Agilent Technologies Inc., USA). The content of unloaded micelles is $m_{3}$. Micelles were achieved using a reversed phase C18 ODS-SP column $(15 \mathrm{~cm} \times 4.6 \mathrm{~mm}$, Inertsil, JAP). The mixture of ethanol/methanol $(1: 1 \mathrm{v} / \mathrm{v})$ was used as the mobile phase at a flow rate of $1 \mathrm{~mL} / \mathrm{min}$ with $\mathrm{UV}$ detection at $275 \mathrm{~nm}$ :

$$
E E \%=\frac{m_{2}}{m_{1}} \times 100 \% ; \quad D L \%=\frac{m_{2}}{m_{1}+m_{3}} \times 100 \%
$$

DSC Analysis. The differential scanning calorimetry (DSC) thermogram of the pure CoQ10, HS15, CoQ10loaded micelles, and physical mixture of CoQ10 and HS15 was performed using a NETZSCH apparatus (DSC200PC, NETZSCH, Germany). Measurements were conducted at a heating rate of $10^{\circ} \mathrm{C} / \mathrm{min}$ from 25 to $350^{\circ} \mathrm{C}$. The thermal behavior was studied by heating about $3 \mathrm{mg}$ of samples in an aluminum pan under nitrogen gas. An empty aluminum pan was utilized as a reference (26).

Determination of $C M C$. CMC was determined by the pyrene fluorescence method with slight modification from the reported method (27). From a stock solution of $5 \times$ $10^{-4} \mathrm{~mol} / \mathrm{L}$ pyrene dissolved in acetone, $0.1 \mathrm{~mL}$ aliquots were transferred with a micropipette into a series of clean, dry test volumetric flasks, and the solvent was allowed to evaporate under nitrogen. A series of unloaded and CoQ10-loaded micellar solutions were added to dry pyrene. Pyrene concentration in each volumetric flask was $5 \times 10^{-6} \mathrm{~mol} / \mathrm{L}$. The mixtures were shaken in dark for $24 \mathrm{~h}$ at $25^{\circ} \mathrm{C}$. The concentration of solubilized pyrene in micellar phase was determined spectrofluorometrically (F7000, Hitachi High-Tech, Japan) at wavelengths of excitation ( $\lambda$ ex) of $336 \mathrm{~nm}$ and emission $(\lambda \mathrm{em})$ of $350 \sim 450 \mathrm{~nm}$.

In Vitro Drug Release. The release of CoQ10 from CoQ10-loaded micelles was inquired by the dialysis bag method (28). A screening for the most suitable release medium was performed. Three milliliters of CoQ10-loaded micelles (amount of CoQ10 was $5 \mathrm{mg}$ ) was placed in a dialysis bag (cutoff 8-12 kDa, Solarbio, USA) and was kept in $150 \mathrm{~mL}$ of release medium at $37^{\circ} \mathrm{C}$, agitated at $100 \mathrm{rpm}$ using an incubating shaker. Two-milliliter samples were drawn at predetermined time intervals, and the same volume was replenished with fresh medium. The amount of released CoQ10 was measured using HPLC. The following release mediums were selected for release studies $(n=3)$ : (i) $0.5 \%$ $(w / v)$ polysorbate 80 ( $\mathrm{pH} 7.4$ phosphate-buffered saline, PBS), (ii) $1.0 \%(w / v)$ polysorbate $80(\mathrm{pH} 7.4 \mathrm{PBS})$, (iii) $15 \%$ ethanol (pH $7.4 \mathrm{PBS}$ ), and (iv) $1.0 \%$ sodium dodecyl sulfate (0.15 M NaCl) (29-31).

\section{Stability (Refrigerated, Accelerated, and Long-Term) Studies}

The thermal stability of CoQ10-loaded micellar samples was monitored under the refrigerated and accelerated conditions for 6 months. They were stored in the closed desiccators with the constant relative humidity $(\mathrm{RH})$ of $75 \pm 5 \%$, maintained by saturated $\mathrm{NaCl}$ solution. The desiccators were maintained at $4 \pm 2$ and $30 \pm 2^{\circ} \mathrm{C}$ respectively in a thermostat without direct light. The appearance, particle size, and PDI were analyzed at the time of 0,3 , and 6 months after treatment.

The long-term stability of CoQ10-loaded micellar samples was also studied at $25 \pm 2^{\circ} \mathrm{C}$ by the similar strategy as described above. The samples were stored in the closed desiccators with the constant relative humidity $(\mathrm{RH})$ of $60 \pm 10 \%$, maintained by saturated $\mathrm{NaNO}_{2}$ solution for $0,3,6,9,12$, and 18 months. The stability of samples were examined, where the appearance, the particle size, PDI, the content of CoQ10, and related substance were indicators $(27,32)$.

\section{RESULTS}

\section{Solubilization by Micelles}

CoQ10 was used as test solute for investigating the solubilization properties of HS15 and polysorbate 80 . This drug is poorly soluble in water. The HS15 was found to be superior in CoQ10 solubilization compared with polysorbate 80 (Fig. 1) which was visualized by the slightly higher slopes in the linear graph of solubilizing system. HS15 solubilized more CoQ10 than polysorbate 80 , which could be explained by the more hydrophobic acyl chain groups in the micellar structure 


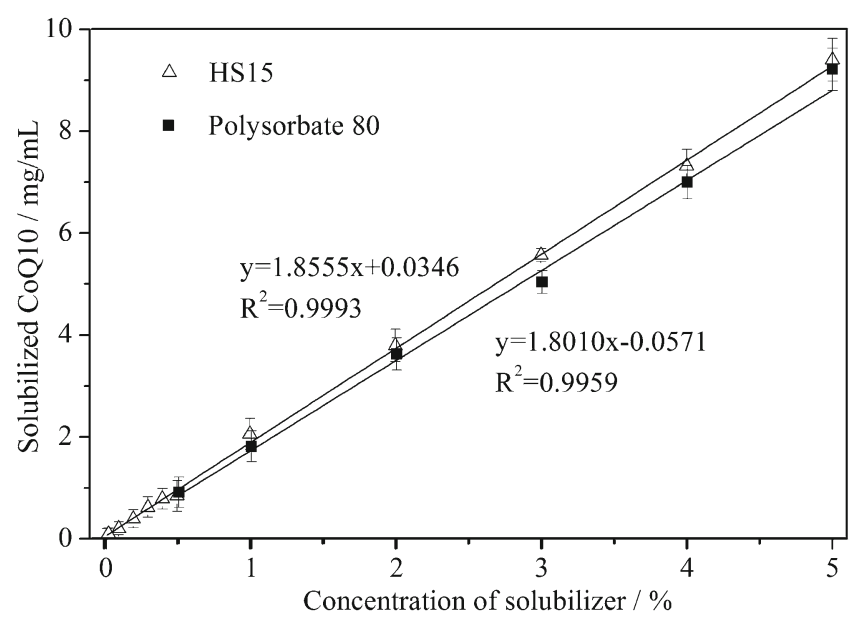

Fig. 1. Solubilization of CoQ10 at increasing HS15 and polysorbate 80 concentrations

(31,33,34). Furthermore, HS15 had been reported to improve the bioavailability and reduce the cytotoxicity (35-37). Accordingly, a low amount of HS15 was used as the vehicle for the production of CoQ10.

\section{The Hemolysis of HS15}

The hemolysis assay was performed to estimate the biocompatibility of the HS15. The level of hemolysis was compared with that of polysorbate 80 , which was a typical surfactant approved by FDA for intravenous administration (38). Hemolysis of HS15 and polysorbate 80 at various times are shown in Fig. 2. HS15 showed much less hemolysis than polysorbate 80 at the concentrations of 0.12 and $0.24 \mathrm{mg} / \mathrm{mL}$. In addition, the hemolysis was 40 and $90 \%$ for HS15 and polysorbate 80 , respectively, while the concentration increased to $0.24 \mathrm{mg} / \mathrm{mL}$ in this study. This indicated that HS15 had much better biocompatibility than polysorbate 80 . It could be noted that as the concentration increased from 0.24 to $0.6 \mathrm{mg} / \mathrm{mL}$, hemolysis induced by HS15 increased dramatically from $44.41 \pm 0.10$ to $99.13 \pm 0.21 \%$, but it was lower than that of polysorbate 80 after $24 \mathrm{~h}$. This indicated that the concentration of HS15 in the micelles should be less than $0.60 \mathrm{mg} / \mathrm{mL}$

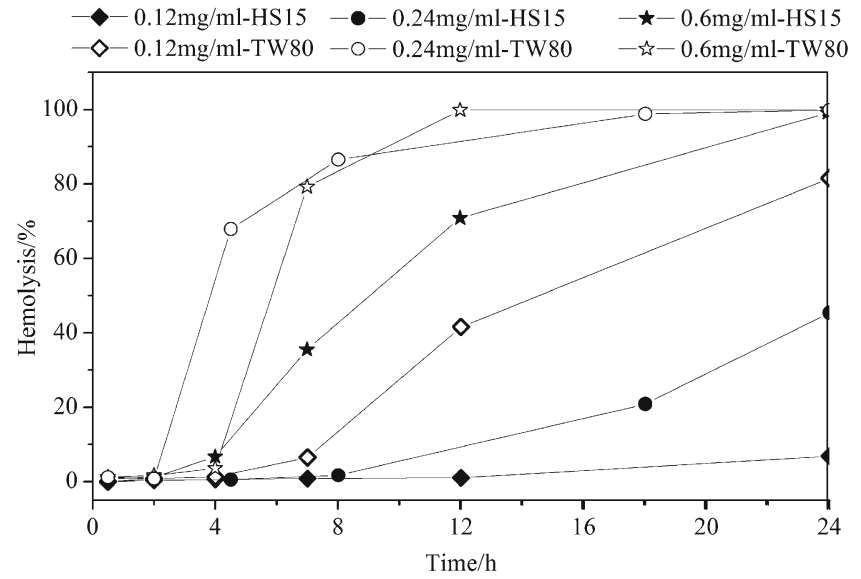

Fig. 2. Hemolysis of HS15 and polysorbate 80 in 24 h (Tw 80: polysorbate 80 ) from the perspective of safety. Hence, HS15 was excellently used as the vehicle for poorly soluble drugs.

\section{Preparation and Optimization of CoQ10-Loaded Micelles}

\section{Effect of the Ratio}

In the present study, the amount of CoQ10 was kept constant (5 mg) and HS15 concentration was varied (i.e., 10, $15,20,30,35$, and $40 \mathrm{mg}$ ). The effect of different weight ratios of CoQ10 and HS15 on the transmittance, particle size, polydispersity index (PDI), entrapment efficiency (EE\%), and drug loading (DL\%) is summarized in Table I. It became evident from the results-an emulsion with large particle size $(>100 \mathrm{~nm})$ and smaller transmittance was obtained when CoQ10 was added alone with HS15 in the ratio of 1:3. This explained by the release of the unencapsulated CoQ10. With increasing of the ratio (from 1:4 to 1:6 ), decreases in the particles size and PDI were obtained. Further, the ratio was varied to $1: 7$ and 1:8 $(w / w)$ for fine tuning the formula, but this led to a significant decrease of DL\%. Hence, the formulation F-5 (1:6) was picked as the optimized ratio for further studies.

\section{Effect of the Stabilizer}

The optimized ratio (1:6) was used to prepare the CoQ10loaded micelles. We used as stabilizers $0.8 \%$ of sodium pyrosulfite, cysteine, thiourea, and glucose (Table II). It showed lower content of the related substance after sterilization compared with that of the others. Hence, glucose was selected in our subsequent formulation. Further, glucose concentrations were varied from 0.05 to $1.0 \%(w / v)$ for fine tuning the formula. Table III shows the effect of different concentrations on the content of CoQ10 and the related substance. Increasing the concentration and storage interval, the content of related substance was weakly charged but led to a significant increase of the content of CoQ10 except for $0.2 \%$. Furthermore, the extremum contents of CoQ10 and the related substance were 97.70 and $0.33 \%$, respectively, at $0.20 \%$ glucose. Hence, $0.2 \%(w / v)$ glucose in the formulation was selected.

\section{Effect of $p H$}

In order to find the optimum preparation method for the CoQ10-loaded micelles, the $\mathrm{pH}$ of the solution was adjusted to $3 \sim 7$ by using $\mathrm{HCl}$ or $\mathrm{NaOH}$. Table IV shows the change of particle size, PDI, zeta potential, and the related substance at different $\mathrm{pH}$ values. It was clear from the data that no significant changes of the particle size, PDI, zeta potential, and CoQ10 content were observed. Nevertheless, the minimum particle size and content of the related substance after sterilization were $18.95 \pm 0.63 \mathrm{~nm}$ and $0.26 \%$, respectively, at $\mathrm{pH} 4$. Hence, $\mathrm{pH} 4$ was selected for further optimization studies.

\section{Effect of Sterilization}

It was shown by the literature (39) that autoclaving was possible in the case of CoQ10 stabilized injection. The CoQ10loaded micellar samples were sterilized at $118^{\circ} \mathrm{C}$ for $25 \mathrm{~min}$ or at $121^{\circ} \mathrm{C}$ for $20 \mathrm{~min}$. After sterilization, the obtained sample 
Table I. Effect of the Ratio of CoQ10 and HS15 on the Transmittance, Particle Size, PDI, EE\%, and DL\%

\begin{tabular}{lccccc}
\hline Batch & Ratio & Transmittance (\%) & Particle size (nm) & PDI & EE\% \\
\hline F-1 & $1: 2$ & 75.5 & $111.70 \pm 1.00$ & $0.223 \pm 0.014$ & - \\
F-2 & $1: 3$ & 75.0 & $127.00 \pm 1.30$ & $0.252 \pm 0.009$ & - \\
F-3 & $1: 4$ & 98.9 & $24.25 \pm 0.56$ & $0.324 \pm 0.008$ & 99.96 \\
F-4 & $1: 5$ & 98.6 & $20.60 \pm 0.46$ & $0.257 \pm 0.028$ & 99.91 \\
F-5 & $1: 6$ & 98.9 & $20.92 \pm 0.43$ & $0.193 \pm 0.009$ & 16.62 \\
F-6 & $1: 7$ & 98.7 & $19.34 \pm 0.23$ & $0.245 \pm 0.028$ & 99.39 \\
F-7 & $1: 8$ & 98.6 & $17.89 \pm 0.15$ & $0.223 \pm 0.024$ & 99.85 \\
\hline
\end{tabular}

$P D I$ polydispersity index, $E E \%$ entrapment efficiency, $D L \%$ drug loading

and the particle size along with PDI were checked by zeta sizer. Different properties of sterilized CoQ10-loaded micellar samples such as the particle size, PDI, the content of CoQ10, and the related substance (before and after sterilization) are given in Table V. In both cases, the particle size and PDI increased slightly after sterilization. The mean CoQ10 contents after sterilization were found to be 98.47 and $91.32 \%$ with the related substance of 0.39 and $4.91 \%$, respectively. This may be due to more degradation of the CoQ10-loaded micellar sample under higher temperature. Hence, $118^{\circ} \mathrm{C}$ for $25 \mathrm{~min}$ was selected for sterilization.

\section{Characterization of Micelles}

\section{The Particle Size Analysis by DLS}

CoQ10-loaded micellar particle size of the sample was measured by DLS. The mean particle size, PDI, and zeta potential of the sample were found to be $19.76 \mathrm{~nm}$ (Fig. 3), 0.112 , and $-3.405 \mathrm{mV}$, respectively, by the optimized method and condition.

\section{$E E \%$ and $D L \%$}

CoQ10 molecule comprises a quinone ring and a long isoprene side chain, which renders it lipophilic and favorable for encapsulation via micelles (40). CoQ10-loaded micelles were in the supernatant, while the unencapsulated CoQ10 likely existed as crystals in the precipitation by centrifugation.
Therefore, it has been proved that unencapsulated CoQ10 was completely removed by centrifugation, so we proceeded to analyze EE\% and DL\%. Nevertheless, the structure of micelles might be disaggregated by centrifugation. The centrifugal speeds of $14,000 \mathrm{rpm}$ for $5 \mathrm{~min}$ was tried in the case of irregular and low "EE\%." With an optimal speed of centrifugation (data not shown), the desired regularity $\mathrm{EE} \%$ was achieved at 12,000 rpm. Hence, 12,000 rpm was selected for further research. Effect of centrifugal time on EE\% and DL\% was studied when the centrifugal speed is $12,000 \mathrm{rpm}$ (Fig. 4). Figure 4 shows a significant reduction in $\mathrm{EE} \%$ with the increasing centrifugal time (0 to $10 \mathrm{~min}, 15$ to $20 \mathrm{~min}$ ), which could be explained by the release of the unencapsulated CoQ10 and disintegration of CoQ10-load micelles, respectively. Thereby, the EE\% and DL\% of CoQ10-loaded micelles were 99.39 and $13.77 \%$, respectively, under centrifugal condition of $12,000 \mathrm{rpm}$ for $10-15 \mathrm{~min}$. They were higher than some polymeric micelles due to the nature of HS15 together with a longer hydrophobic block.

\section{Differential Scanning Calorimetry}

DSC was performed to further test the physical states of CoQ10-loaded micelles. The DSC thermograms of CoQ10loaded micelles, HS15, CoQ10, and physical mixture are shown in Fig. 5. The DSC curves of pure CoQ10 and HS15 exhibited endothermic peaks around 54 and $30^{\circ} \mathrm{C}$, respectively, which corresponded to their intrinsic melting points. CoQ10-HS15 micelles did not show any melting peak of drug or HS15,

Table II. Effect of Type of Stabilizer on the Content of CoQ10 and the Related Substance

\begin{tabular}{|c|c|c|c|c|c|c|}
\hline \multirow[t]{2}{*}{ Type } & \multirow[t]{2}{*}{ Content $(\%)$} & \multirow[t]{2}{*}{ Without sterilization } & \multicolumn{4}{|c|}{ With sterilization, storage interval (week) } \\
\hline & & & 0 & 1 & 2 & 3 \\
\hline \multirow[t]{2}{*}{ Sodium metabisulfite } & CoQ10 & 97.89 & 97.31 & 96.97 & 94.97 & 90.22 \\
\hline & The related substance & 0.49 & 0.66 & 0.61 & 0.75 & 0.82 \\
\hline \multirow[t]{2}{*}{ Cysteine } & CoQ10 & 98.70 & 95.96 & 94.76 & - & - \\
\hline & The related substance & 0.47 & 0.98 & 1.23 & - & - \\
\hline \multirow[t]{2}{*}{ Thiourea } & CoQ10 & 100.00 & 98.65 & 95.41 & 95.59 & 95.04 \\
\hline & The related substance & 0.39 & 0.53 & 0.70 & 0.73 & 0.79 \\
\hline \multirow[t]{2}{*}{ Glucose } & CoQ10 & 99.17 & 98.54 & 98.54 & 98.01 & 97.90 \\
\hline & The related substance & 0.32 & 0.32 & 0.40 & 0.50 & 0.54 \\
\hline
\end{tabular}

“-” unmeasured

CoQ10 coenzyme Q10 
Table III. Effect of Concentration of Glucose on the Content of CoQ10 and the Related Substance

\begin{tabular}{|c|c|c|c|c|c|c|}
\hline \multirow[t]{2}{*}{ Content $(\%)$} & \multirow[t]{2}{*}{ Concentration of glucose (\%) } & \multirow[t]{2}{*}{ Without sterilization } & \multicolumn{4}{|c|}{ With sterilization, storage interval (week) } \\
\hline & & & 0 & 1 & 2 & 3 \\
\hline \multirow[t]{5}{*}{ CoQ10 } & 0.05 & 99.54 & 98.20 & 97.73 & 97.60 & 97.32 \\
\hline & 0.10 & 99.61 & 99.39 & 98.44 & 97.97 & 97.62 \\
\hline & 0.20 & 99.55 & 98.83 & 97.63 & 96.91 & 97.70 \\
\hline & 0.50 & 99.83 & 99.02 & 98.29 & 96.27 & 96.19 \\
\hline & 1.00 & 100.00 & 98.03 & 98.46 & 97.76 & 97.71 \\
\hline \multirow[t]{5}{*}{ The related substance } & 0.05 & 0.15 & 0.35 & 0.38 & 0.43 & 0.52 \\
\hline & 0.10 & 0.21 & 0.35 & 0.42 & 0.44 & 0.56 \\
\hline & 0.20 & 0.17 & 0.33 & 0.31 & 0.33 & 0.33 \\
\hline & 0.50 & 0.19 & 0.32 & 0.30 & 0.42 & 0.43 \\
\hline & 1.00 & 0.28 & 0.29 & 0.40 & 0.39 & 0.45 \\
\hline
\end{tabular}

CoQ10 coenzyme Q10

indicating that CoQ10 might have dispersed into molecular form in the carrier. On the contrary, the physical mixture indicated the melting peak of CoQ10 and HS15. Taken together, these DSC profiles demonstrated that the CoQ10-HS15 micelles, composed of HS15 and CoQ10, were not a simple physical mixture of their individual component.

\section{$C M C$}

As an important fluorescence probe with long fluorescent lifetime, pyrene has enough sensitivity to the change of polarity. CMC could be calculated at the point where the $I_{373} / I_{384}$ ratio increases dramatically (41). The graphs for CMC determination of unloaded and CoQ10-loaded micelles are provided in Fig 6. The CMC of unloaded micelles was $5.411 \times 10^{-5} \mathrm{~g} /$ $\mathrm{mL}$, which was lower than that of other nonionic surfactants. This result suggested that HS15 could be utilized as an effective means to stabilize chemically unstable drugs as well as to solubilize poorly soluble drug. Significantly, the CMC of CoQ10-loaded micelles was $5.623 \times 10^{-4} \mathrm{~g} / \mathrm{mL}$, describing excellent thermodynamic stability of the drug and integrity toward dilution, which was in favor of reducing or avoiding exposure.

\section{In Vitro Drug Release Study}

To obtain the best release model and mechanism of CoQ10-loaded micelles, the drug release profile has been further explored. The minimum solubility of CoQ10 in the medium was $0.17 \mathrm{mg} / \mathrm{mL}$ which satisfied the sink condition $(>0.1 \mathrm{mg} / \mathrm{mL})$. Figure 7 shows that the highest percentage of released CoQ10 was obtained in $1.0 \%$ polysorbate $80, \mathrm{pH} 7.4$, PBS. In addition, the drug released from micelles started with an initial burst in the first $22.5 \mathrm{~h}$ and was followed by a stably sustained release behavior. The cumulative percent of drug released for over $72 \mathrm{~h}$ was only $4 \%$. The data of the release over $72 \mathrm{~h}$ were fitted using three equations, zero order, first order, and Higuchi equation (Table VI). The obtained results from the kinetic studies are listed in Table VI. These data suggested that the first order was the best fit kinetic model with $r^{2}$ value of 0.9843 and 0.9978 .

\section{THERMAL STABILITY}

The thermal stability of a promising drug candidate plays an important role in the process of drug development. Figure 8 shows the changes in particle size and PDI during the storage time at different temperatures. Upon both $4^{\circ} \mathrm{C}$ and room temperature, all samples remained more stable compared to those at $30^{\circ} \mathrm{C}$. A significant increase in their particle size at $30^{\circ} \mathrm{C}$ was observed after six months (about $100 \mathrm{~nm}$ ), and their PDI was up to 0.4 , which reflected relatively homogeneous micelles and indicated that the micelles were sensitive to temperature (42).

To identify the storage conditions and handling requirements for the samples and ensure drug quality within the

Table IV. Effect of $\mathrm{pH}$ on the Particle Size, PDI, Zeta Potential, the Content of CoQ10, and the Related Substance

\begin{tabular}{|c|c|c|c|c|c|c|c|}
\hline \multirow[t]{2}{*}{$\mathrm{pH}$ value } & \multirow{2}{*}{$\begin{array}{l}\text { Particle size } \\
\quad(\mathrm{nm})\end{array}$} & \multirow[t]{2}{*}{ PDI } & \multirow{2}{*}{$\begin{array}{l}\text { Zeta potential } \\
\qquad(\mathrm{mV})\end{array}$} & \multicolumn{2}{|c|}{ Without sterilization } & \multicolumn{2}{|c|}{ After sterilization } \\
\hline & & & & $\begin{array}{c}\text { The content of } \\
\text { CoQ10 }(\%)\end{array}$ & $\begin{array}{l}\text { The content of } \\
\text { related } \\
\text { substance }(\%)\end{array}$ & $\begin{array}{c}\text { The content of } \\
\text { CoQ10 }(\%)\end{array}$ & $\begin{array}{l}\text { The content of related } \\
\text { substance }(\%)\end{array}$ \\
\hline 3 & $20.65 \pm 0.38$ & $0.152 \pm 0.011$ & $-2.19 \pm 0.827$ & 99.10 & 0.26 & 98.83 & 0.34 \\
\hline 4 & $18.95 \pm 0.63$ & $0.140 \pm 0.037$ & $-2.66 \pm 0.233$ & & & 98.36 & 0.28 \\
\hline 5 & $19.85 \pm 0.70$ & $0.167 \pm 0.030$ & $-2.44 \pm 0.205$ & & & 97.80 & 0.39 \\
\hline 6 & $19.68 \pm 0.39$ & $0.218 \pm 0.005$ & $-4.36 \pm 0.403$ & & & 98.73 & 0.41 \\
\hline 7 & $19.42 \pm 0.22$ & $0.175 \pm 0.019$ & $-4.46 \pm 0.339$ & & & 98.24 & 0.44 \\
\hline
\end{tabular}


Table V. Effect of Sterilization on the Particle Size, PDI, Zeta Potential, the Content of CoQ10, and the Related Substance

\begin{tabular}{lccccc}
\hline \multicolumn{1}{c}{ Condition } & $\begin{array}{c}\text { Particle size } \\
(\mathrm{nm})\end{array}$ & 3PDI & $\begin{array}{c}\text { Zeta potential } \\
(\mathrm{mV})\end{array}$ & $\begin{array}{c}\text { The content of } \\
\text { CoQ10 }(\%)\end{array}$ & $\begin{array}{c}\text { The content of related } \\
\text { substance }(\%)\end{array}$ \\
\hline Before sterilization & $18.73 \pm 0.27$ & $0.082 \pm 0.027$ & $-1.46 \pm 0.29$ & 99.27 & 0.38 \\
$118^{\circ} \mathrm{C}, 25 \mathrm{~min}$ & $19.76 \pm 0.13$ & $0.112 \pm 0.007$ & $-3.41 \pm 1.24$ & 98.47 & 0.39 \\
$121^{\circ} \mathrm{C}, 20 \mathrm{~min}$ & $19.56 \pm 0.03$ & $0.101 \pm 0.012$ & $-2.54 \pm 0.25$ & 91.32 & 4.91 \\
\hline
\end{tabular}

PDI polydispersity index, CoQ10 coenzyme Q10

recommended period of validity, three primary batches of samples were further investigated on their stability under accelerated $\left(30^{\circ} \mathrm{C}\right.$ for 6 months $)$ and long-term $\left(25^{\circ} \mathrm{C}\right.$ for 18 months) conditions. Apparently, $\mathrm{pH}$, the content of CoQ10, and the related substance of the samples were examined. The assay results are summarized in Table VII. There was no noticeable change on the appearance of sample in the accelerated and long-term stability investigations (data not shown). There was a slight change on $\mathrm{pH}$ value of samples. The HPLC results also indicated that the content of CoQ10 and the related substance at 6-month testing periods had more noticeable change as compared to that at 18 -month testing periods; in the end, they were charged to 92.93 and $0.74 \%$, respectively. Hence, the CoQ10-loaded micelles had excellent stability below $25^{\circ} \mathrm{C}$. Figure 9 shows the regression assay of a drug product with upper and lower acceptance criteria of 105 and $95 \%$ of label claim, respectively, according to the regulation of the International Conference of Harmonization (ICH) (40). The lower confidence limit of $95 \%$ intersects the lower acceptance criterion of content of CoQ10 at 31.2 months, while the upper confidence limit did not intersect with the upper acceptance criterion until later. The upper one-sided $95 \%$ confidence limit for the mean intersects the acceptance criterion of the content of the related substance when it exceeds 36 months. Therefore, the proposed shelf of 24 months can be supported by the statistical analysis of the data.

\section{DISCUSSION}

In solubility study, a systematic linear increase in CoQ10 solubility with an increasing concentration of HS15 in water

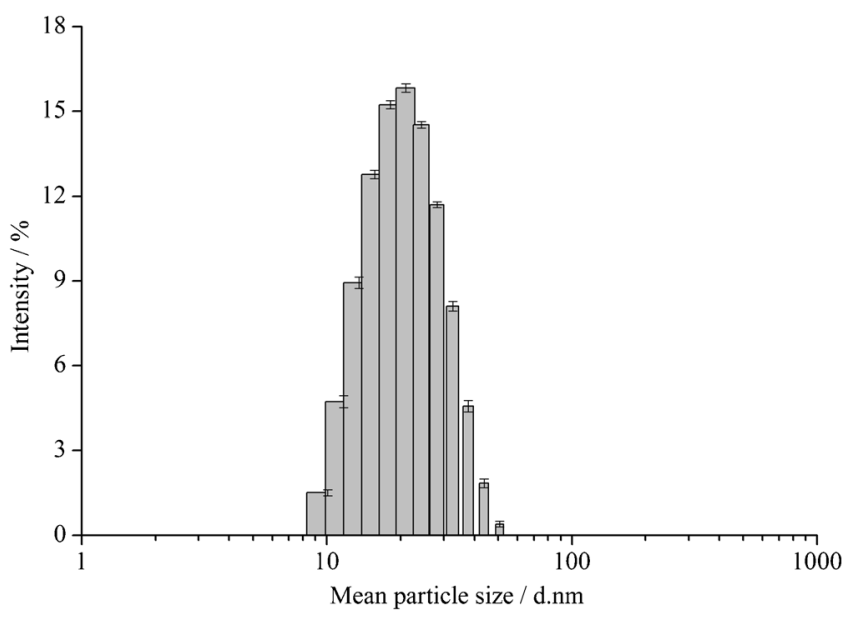

Fig. 3. Micellar size distribution of CoQ10-loaded HS15 might be attributed to the improved wettability and dissolution of CoQ10 particles in water by HS15 (8). An indication of the process of transferring CoQ10 from pure water to the aqueous solutions of HS15 was obtained from the change of Gibbs free energy. The Gibbs free energy of transferring $\left(\Delta G^{\mathrm{O}}{ }_{\mathrm{tr}}\right) \mathrm{CoQ} 10$ from pure water to the aqueous solutions of HS15 was calculated using Eq. 1 , where $S_{\mathrm{o}} / S_{\mathrm{s}}$ is the ratio of molar solubility of CoQ10 in aqueous solutions of HS15 to that of the pure water. The ratio of $S_{\mathrm{o}}$ and $S_{\mathrm{s}}$ is more than 1 , so that $\Delta G^{\mathrm{O}}{ }_{\mathrm{tr}}$ values were all negative indicating the spontaneous nature of CoQ10 solubilization, and they decreased with an increment in HS15 concentration which demonstrated that the reaction became more favorable as the concentration of HS15 increased.

$\Delta G^{\mathrm{O}}{ }_{t \mathrm{r}}=-2.303 R T \log \left(\mathrm{S}_{\mathrm{O}} / \mathrm{S}_{\mathrm{S}}\right)$

CoQ10-loaded micelles were prepared by direct dissolution method. It was various process variables like micellar compositions, the preparation condition, and the preparation method that impacted micelles. Formulation F-5 with drug HS15 ratio 1:6 was selected as the optimized ratio because it gave lower particle size, higher entrapment efficiency, and higher drug loading capacity. This might be attributed to the presence of CMC of the CoQ10-loaded micelle which was confirmed by fluorescence probe measurement and was measured to be $5.623 \times 10^{-4} \mathrm{~g} / \mathrm{mL}$. The concentration of formulation F-4 was $4.0 \times 10^{-4} \mathrm{~g} / \mathrm{mL}$ which approached CMC, so that the transmittance of formulation F-4 was much higher than that of formulations F-1, F-2, and F-3. Hence, formulation F-5

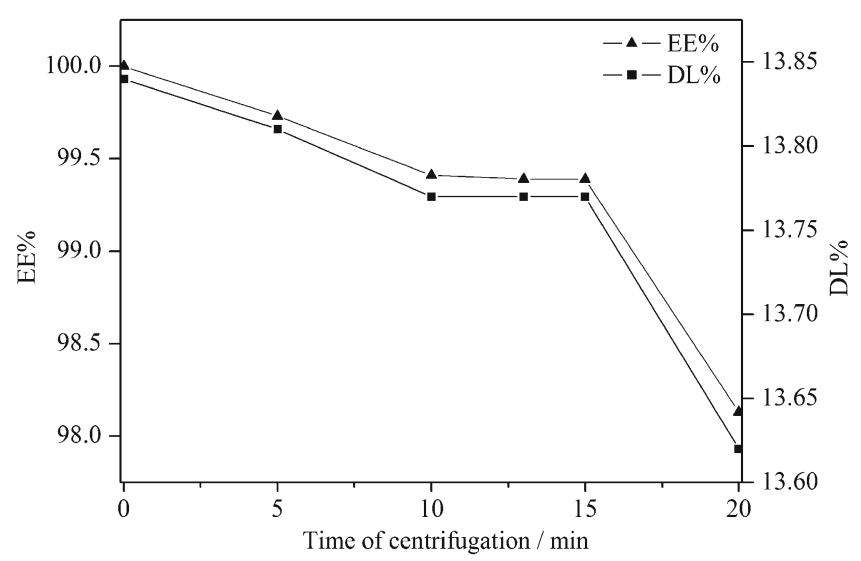

Fig. 4. Effect of centrifugal time on loading content and entrapment efficiency. The centrifugal speed is $12,000 \mathrm{rpm}$ 


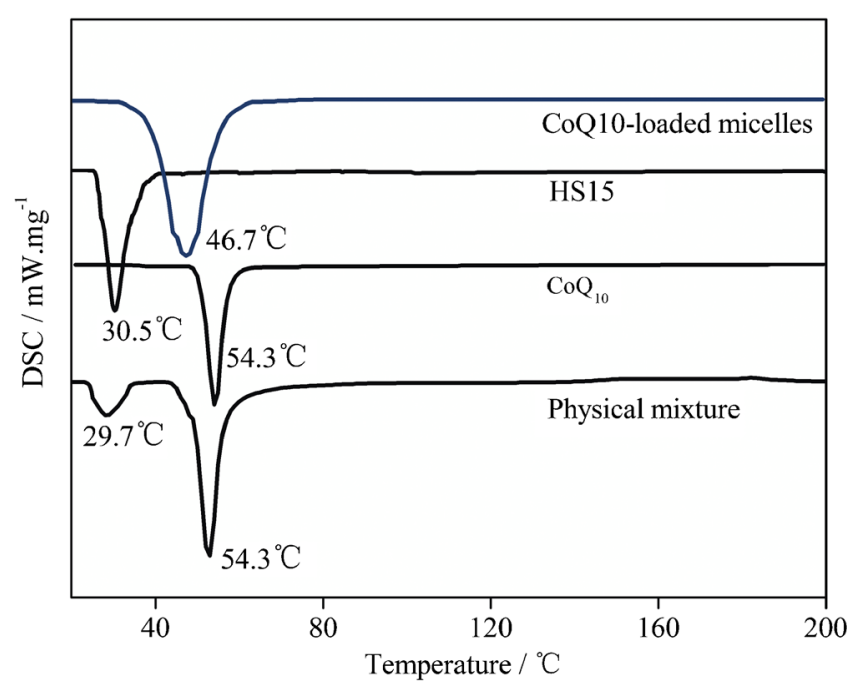

Fig. 5. Overlay of DSC thermograms of $\mathrm{CoQ}_{10}$-loaded micelles, HS $15, \mathrm{CoQ}_{10}$, and physical mixture. (Physical mixture: physical mixture of CoQ10 and HS 15)

was selected as optimized one and taken for further studies concerning $\mathrm{pH}$ value, the concentration of stabilizer, and the sterilization condition.

Glucose $(0.20 \%)$ was found to boast better stability after sterilization with the content of CoQ10 and the related substance standing at 97.70 and $0.33 \%$, respectively. Apart from the above formulation benefits, it was hypothesized that the regular water structure imparted by glucose would assist in enhancing the stability of micelle (43). Optimizing the $\mathrm{pH}$ value did not change significantly at particle size, PDI, and zeta potential, but interestingly, stability showed lower content of the related substance (Table IV) due to the microenvironment which was a major factor of micellar stability (14). That could be attributed to enhancing the drug-core interaction when $\mathrm{pH}$ value was 4 .

The fate of the micelles after injection was mainly influenced by particle size, PDI, and zeta potential. They were important to the micelles for their recognition or nonrecogni-

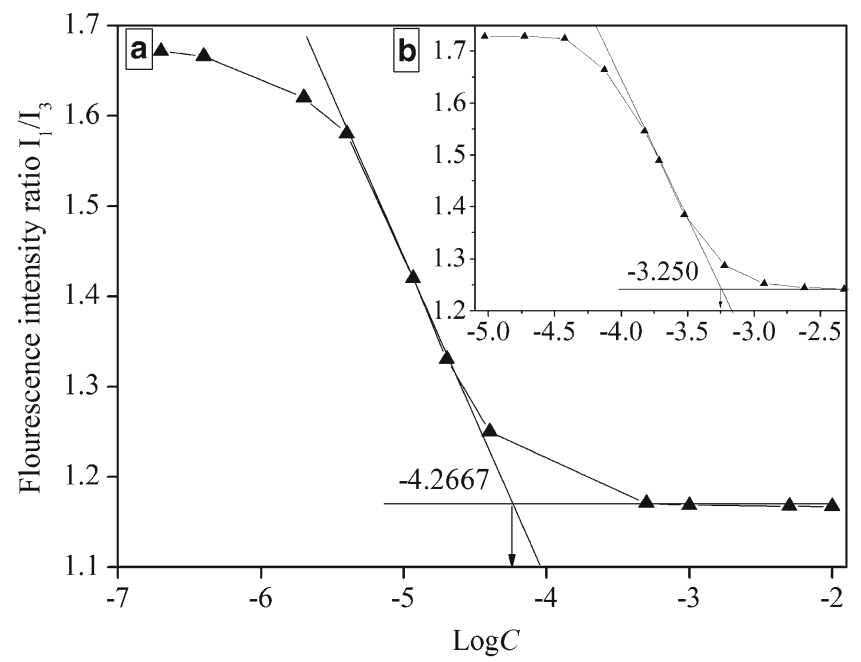

Fig. 6. Fluorescence intensity ratios of $I_{373} / I_{384}$ versus the logarithm of different HS15 and CoQ10-loaded micellar concentration ( $a$ : unloaded micelles; $b$ : CoQ10-loaded micelles)
Table VI. Release Kinetics of CoQ10-Loaded Micelles in $1 \%$ of Polysorbate 80

\begin{tabular}{|c|c|c|c|}
\hline Time/h & Release kinetics & Equation & $r^{2}$ \\
\hline \multirow[t]{3}{*}{$0-22.5$} & Zero order & $R=0.1602 t+0.2981$ & 0.9833 \\
\hline & First order & $\begin{array}{l}\ln (100-R)=-0.0016 t \\
-0.0029\end{array}$ & 0.9843 \\
\hline & Higuchi equation & $R=0.7965 t^{1 / 2}-0.2450$ & 0.9826 \\
\hline \multirow[t]{3}{*}{$22.5-72.0$} & Zero order & $R=0.0046 t+3.7169$ & 0.9978 \\
\hline & First order & $\begin{array}{l}\ln (100-R)=0.04115 t \\
-0.0379\end{array}$ & 0.9978 \\
\hline & Higuchi equation & $R=0.0612 t^{1 / 2}+3.5204$ & 0.9898 \\
\hline
\end{tabular}

$R=$ percentage of released CoQ10; $t=$ the time of release study

tion by the defense mechanism of body (44). The optimized CoQ10-loaded micelles showed a mean particle size of $19.76 \mathrm{~nm}$. Homogeneous and small particle sizes $(<200 \mathrm{~nm})$ could reduce the uptake of the reticuloendothelial system (40). Furthermore, it was expected to enhance the circulation time in blood which in turn could improve the site-specific targeting (38). The mean particle size of blank micelle was $12.41 \mathrm{~nm}$ which was smaller than the CoQ10-loaded micelles. One can deduce from those findings that the HS15 was not only incorporated within the micellar core but also possibly was integrated into the micellar shell or was adsorbed on the outer shell leading to transformed micelles (23). On one hand, micelles with greater positive or negative zeta potential values produced greater repulsive forces (39). On the other hand, zeta potential of the micelles significantly influenced the stability of drug in blood; specifically, micelles with lower positive or negative zeta potential values reduced its absorption toward albumin and platelet (40), which also improved the site specific targeting.

The initial fast release phase in Fig. 7 could be attributed to the hydration of the interfacial drug molecules and their passive diffusion. The slow (sustained) release was primarily due to the slow diffusion of drug and the erosion or swelling of micelles as shown in literature $(27,45)$. Common drug release profiles from nanoparticles (with surfactants present) exhibited a short and considerable "burst"

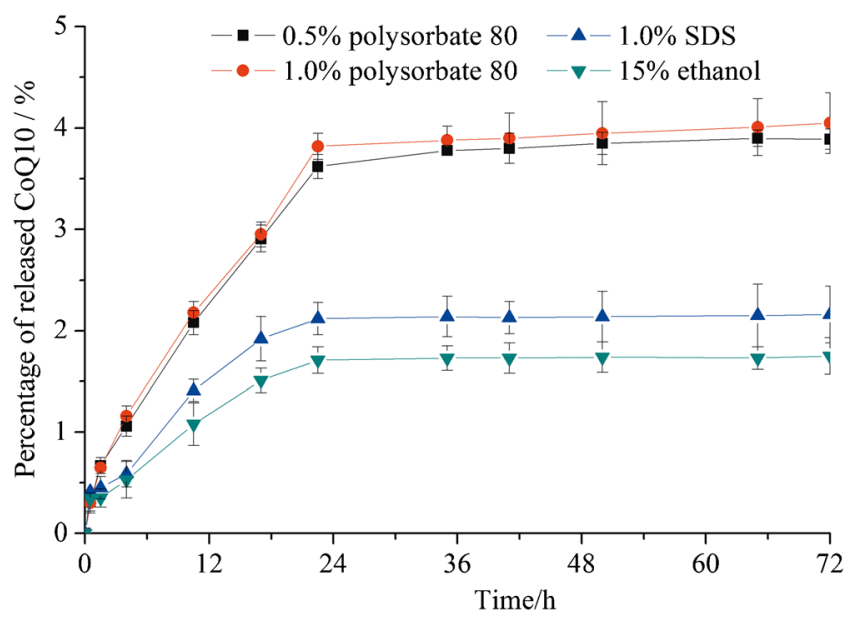

Fig. 7. Release profiles of CoQ10 from CoQ10-loaded micelles in comparison release medium 


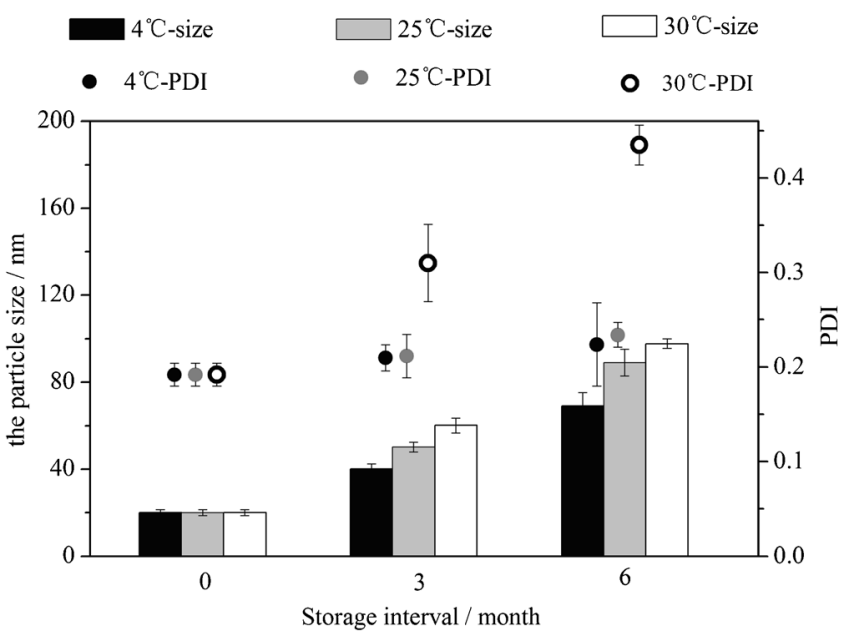

Fig. 8. The particle size and PDI profiles of CoQ10-loaded micelles in different temperatures

lasting 1-2 days followed by slower, but nearly $100 \%$ drug release. Our CoQ10-loaded micelles exhibited a similar profile with an important difference, which resulted from the interactions between the chains in the micellar core and the drug preventing $100 \%$ drug release $(7,9,31,39,42)$. Furthermore, the spatial position of a certain drug within a micelle will depend on its polarity. In aqueous systems, nonpolar molecules will be solubilized within the micelle core (46). CoQ10 is a nonpolar molecule, so that CoQ10 in the micelle core compartment. In addition, an increase in the length of a hydrophobic region of surfactant facilitates the solubilization of the hydrophobic drug inside the micelle core (46). HS15 is a nonionic surfactant, and its molecular structure contains long stearic acid chain. We suspect that that the drug release may also be influenced by spatial position of a certain drug. This slow burst and possibility of biologically mediated CoQ10 release were exciting indicators for sustained antioxidant drug delivery.

Table VII. Accelerated and Long-Term Stability Results of CoQ10Loaded Micelles

\begin{tabular}{lccc}
\hline $\begin{array}{l}\text { Storage interval } \\
\text { (month) }\end{array}$ & $\mathrm{pH}$ & \multicolumn{2}{c}{ Content (\%) } \\
\cline { 3 - 4 } & & CoQ10 & $\begin{array}{l}\text { Related } \\
\text { substance }\end{array}$ \\
\hline Initial & & & $0.20 \pm 0.01$ \\
Accelerated stability & & & \\
$\left(6\right.$ months, $\left.30 \pm 2^{\circ} \mathrm{C}\right)$ & & & \\
3 & $3.99 \pm 0.04$ & & \\
6 & $3.85 \pm 0.07$ & $94.78 \pm 0.55$ & $0.53 \pm 0.00$ \\
Long-term stability & & $92.93 \pm 0.42$ & $0.74 \pm 0.03$ \\
$\left(18\right.$ months, $\left.25 \pm 2^{\circ} \mathrm{C}\right)$ & & & \\
3 & $3.95 \pm 0.04$ & $99.71 \pm 0.61$ & $0.24 \pm 0.01$ \\
6 & $3.98 \pm 0.03$ & $99.08 \pm 0.64$ & $0.31 \pm 0.02$ \\
9 & $3.97 \pm 0.03$ & $98.60 \pm 0.56$ & $0.37 \pm 0.02$ \\
12 & $4.02 \pm 0.03$ & $98.13 \pm 0.52$ & $0.44 \pm 0.02$ \\
18 & $3.98 \pm 0.02$ & $97.37 \pm 0.67$ & $0.69 \pm 0.01$ \\
\hline
\end{tabular}

CoQ10 coenzyme Q10

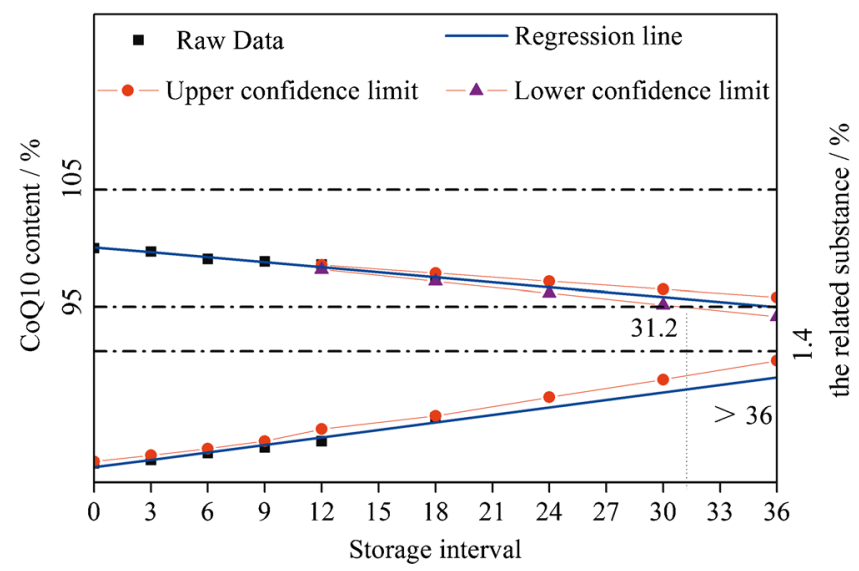

Fig. 9. Shelf life estimation with upper and lower acceptance criteria based on assay at $25^{\circ} \mathrm{C}$

The data of thermal stability study revealed the stability of CoQ10-loaded micelle samples was fine, while the marketed formulation $\mathrm{Hefu}{ }^{\circledR}$ was beneficial to crystallization. This could be explained by difference of structure between HS15 and polysorbate 80, and the former has more hydrophobic acyl chain groups than the latter. Owen et al. indicated the stability was directly related to the hydrophobic character of the core, which influenced the drug-core interactions $(14,47)$.

\section{CONCLUSION}

In the present study, a new surfactant HS15 was investigated as a component of colloidal drug carrier system for CoQ10. Different ratios of HS15/CoQ10, $\mathrm{pH}$ value, concentration of glucose, and condition of sterilization were investigated to form a micellar system. It was proved that the concentration of HS15 influenced the particle size, PDI, entrapment efficiency, and drug loading capacity. Furthermore, the $\mathrm{pH}$ value and the related substance content were closely linked. The concentration of glucose and the sterilization condition also effects on the stability. In summary, all these characterizations data support the notion that CoQ10-loaded micelle is a useful system for the effect of CoQ10 on cardiovascular health. The osmotic pressure of CoQ10-loaded micelle is $285 \mathrm{mOsmol} / \mathrm{kg}$ which suggests that it has good isotonicity. The shelf life of the CoQ10-loaded micellar system can be predicted as 24 months based on the storage stability results. Again, this method could be easily scaled up for commercial purpose. Hence, the results of this study showed that HS15 micelle can be applied as drug carrier systems for many poorly soluble or nonsoluble drugs, especially for CoQ10.

\section{ACKNOWLEDGMENTS}

This work has been supported by Chongqing Engineering Research Center for Pharmaceutical Process and Quality Control Capacity building project (CSTC2012gg-yyjsb1000233) and Southwest University Dr. Fund projects (SWU110056, SWU110057) and Chongqing Postgraduate Education Reform researching project (yjg143085). 


\section{REFERENCES}

1. Bonakdar RA, Guarneri E. Coenzyme Q10. Am Fam Physician. 2005;72:1065-70.

2. Ebadi M, Muralikrishnan D, Pellett LJ, et al. Ubiquinone (coenzyme Q10) and complex I in mitochondrial oxidative disorder of Parkinson's disease. Proc West Pharmacol Soc. 2000;43:55-63.

3. Aberg F, Appelkvist EL, Broijersen A, et al. Gemfibrozil-induced decrease in serum ubiquinone and $\alpha$ - and $\gamma$-tocopherol levels in men with combined hyperlipidaemia. Eur J Clin Investig. 1998;28:235-42.

4. Al Gadban MM, Smith KJ, Soodavar F, et al. Differential trafficking of oxidized LDL and oxidized LDL immune complexes in macrophages: impact on oxidative stress. PLoS One. 2010;5:1-10.

5. Turunen M, Olsson J, Dallner G. Metabolism and function of coenzyme Q. BBA-Biomembr. 2004;1600:171-99.

6. Hsu $\mathrm{CH}$, Cui ZR, et al. Preparation and characterization of novel coenzyme Q10 Nanoparticles engineered from microemulsion precursors. AAPS PharmSciTech. 2003;4:1-12.

7. Shin JY, Shin JI, Kim JS, et al. Assembly of Coenzyme Q10 nanostructure resembling nascent discoidal high density lipoprotein particle. Biochem Biophys Res Commun. 2009;388:217-21.

8. Bhandari KH, Newa M, Kim JA, et al. Preparation, characterization and evaluation of coenzyme Q10 binary solid dispersions for enhanced solubility and dissolution. Biol Pharm Bull. 2007:30:1171-6.

9. Nehilla BJ, Bergkvist M, Popat KC, et al. Purified and surfactantfree coenzyme Q10-loaded biodegradable nanoparticles. Int J Pharm. 2008;348:107-14.

10. Swarnakar NK, Jain AK, Singh RP, et al. Oral bioava ilability, therapeutic efficacy and reactive oxygen species scavenging properties of coenzyme Q10-loaded polymeric nanoparticles. Biomaterials. 2011;32:6860-74.

11. Kwon SS, Nam YS, Lee JS, et al. Preparation and characterization of coenzyme Q10-loaded PMMA nanoparticles by a new emulsification process based on microfluidization. Colloid Surf A. 2002;210:95-104.

12. Ray R, Kibbe AH, Rowe R, et al. Handbook of pharmaceutical excipients. Washington, DC: APhA Publications; 2003.

13. Barratt GM. Therapeutic applications of colloidal drug carriers. Pharm Sci Technol Today. 2000;3:163-71.

14. Owen SC, Chan D, Shoichet MS. Polymeric micelle stability. Nano Today. 2012;7:53-65.

15. Ten Tije AJ, Verweij J, Loos WJ, et al. Pharmacological effects of formulation vehicles: implications for cancer chemotherapy. Clin Pharmacokinet. 2003;42:665-85.

16. Coon JS, Knudson W, Clodfelter K, et al. Solutol HS15, nontoxic polyoxyethylene esters of 12-hydroxystearic acid, reverses multidrug resistance. Cancer Res. 1991;51:897-902.

17. Ku S, Velagaleti R. Solutol HS15 as a novel excipient, Pharm Technol. 2010. Solutol HS15 as a novel excipient, Pharm Technol. Accessed 2 Nov 2010.

18. Alani Adam WG, Rao DA, Seidel R, et al. The effect of novel surfactants and solutol ${ }^{\circledR}$ HS15 on paclitaxel aqueous solubility and permeability across a caco-2 monolayer. J Pharm Sci. 2010;99:3473-85.

19. Seo SW, Han HK, Chun MK, et al. Preparation and pharmacokinetic evaluation of curcumin solid dispersion using Solutol® HS15 as a carrier. Int J Pharm. 2012;424:18-25.

20. Ahmad J, Mir SR, Kohli K, et al. Effect of oil and co-surfactant on the formation of Solutol HS15 based colloidal drug carrier by Box-Behnken statistical design. Colloid Surf A. 2014;453:68-77.

21. Bittner B, Gonzalez RCB, Walter I, et al. Impact of Solutol HS15 on the pharmacokinetic behaviour of colchicine upon intravenous administration to male wistar rats. Biopharm Drug Dispos. 2003;24:173-81.

22. Cornaire G, Woodley J, Hermann P, et al. Impact of excipients on the absorption of P-glycoprotein substrates in vitro and in vivo. Int J Pharm. 2004;278:119-31.

23. Rupp C, Steckel H, Mueller BW. Solubilization of poorly watersoluble drugs by mixed micelles based on hydrogenated phosphatidylcholine. Int J Pharm. 2010;395:272-80.
24. Qiu LP, Qiao MX, et al. Enhanced effect of $\mathrm{pH}$-sensitive mixed copolymer micelles for overcoming multidrug resistance of doxorubicin. Biomaterials. 2014;35:9877-87.

25. Wang CT, Ji BQ, Cao YP, et al. Preparation and evaluation of lipid nanoparticles for loading enzyme subtilisin FS3. Food Sci. 2008;29:158-61.

26. Yang M, Liu L, Su QJ, et al. Preparation and characterization of creatine phosphate sodium. Lat Am J Pharm. 2014;33:658-65.

27. Sezgin Z, Yuksel N, Baykara T. Preparation and characterization of polymeric micelles for solubilization of poorly soluble anticancer drugs. Eur J Pharm Biopharm. 2006;64:261-8.

28. Zhai YG, Guo SS, Liu CH, et al. Preparation and in vitro evaluation of apigenin-loaded polymeric micelles. Colloid Surf A. 2013;429:24-30.

29. Tsai M, Lu Z, Wientjes MG, et al. Paclitaxel-loaded polymeric microparticles: quantitative relationships between in vitro drug release rate and in vivo pharmacodynamics. J Control Release. 2013;172:737-44.

30. Crison JR, Weiner ND, Amidon GL. Dissolution media for in vitro testing of water-insoluble drugs: effect of surfactant purity and electrolyte on in vitro dissolution of carbamazepine in aqueous solutions of sodium lauryl sulfate. J Pharm Sci. 1997;86:384-8.

31. Bao YM, Mo XP, Xu XY. Stability studies of anticancer agent bis (4-fluorobenzyl) trisulfide and synthesis of related substances. J Pharm Biomed. 2008;48:664-71.

32. El-Laithy HM. Preparation and physicochemical characterization of dioctyl sodium sulfosuccinate (Aerosol OT) microemul sion for oral drug delivery. AAPS PharmSciTech. 2003;4:1-10.

33. Liu TQ, Gao XG. Properties of epigallocatechin-3-Gallate in Tween80 micelle. Acta Phys -Chim Sin. 2010;26:66-72.

34. Illum L, Jordan F, Lewis AL. CriticalSorb ${ }^{\mathrm{TM}}$ : a novel efficient nasal delivery system for human growth hormone based on Solutol HS15. J Control Release. 2012;162:194-200.

35. Jun LH, Xia YQ, Li D, Zhong LJ, et al. Phospholipid-Tween 80 mixed micelles as an intravenous delivery carrier for paclitaxel. Drug Dev Ind Pharm. 2011;37:597-605.

36. Liu H, Ma XQ. Coenzyme Q10 intravenous infusion and its preparation method: China. 2006. CN200410022007X.

37. Dabholkar RD, Sawant RM, Mongayt DA, et al. Polyethylene glycol-phosphatidylethanolamine conjugate (PEG-PE)-based mixed micelles: some properties, loading with paclitaxel, and modulation of P-glycoprotein-mediated efflux. Int J Pharm. 2006:315:148-57.

38. Win KY, Feng SS. Effects of particle size and surface coating on cellular uptake of polymeric nanoparticles for oral delivery of anticancer drugs. Biomaterials. 2005;26:2713-22.

39. Mitri K, Shegokar R, Gohla S, et al. Lipid nanocarriers for dermal delivery of lutein: preparation, characterization, stability and performance. Int J Pharm. 2011;414:267-75.

40. Chen DW, Yan L, Qiao MX, et al. Preparation of docetaxel loaded $\mathrm{pH}$-sensitive block copolymer micelles. Acta Pharm Sin. 2008:43:1066-70

41. Aguiar J, Carpena P, Molina-Bolívar JA, et al. On the determination of the critical micelle concentration by the pyrene $1: 3$ ratio method. J Colloid Interface Sci. 2003:258:116-22.

42. Wichit A, Tangsumranjit A, et al. Polymeric micelles of PEG-PE as carriers of all-trans retinoic acid for stability improvement. AAPS PharmSciTech. 2012;13:336-43.

43. Oro JRD. Role of co-solute in biomolecular stability: glucose, urea and the water structure. J Biol Phys. 2001;27:73-9.

44. Wilson B, Lavanya Y, Priyadarshini RAB, et al. Albumin nanoparticles for the delivery of gabapentin: preparation, characterization and pharmacodynamic studies. Int J Pharm. 2014;473:73-9.

45. Venkateswarlu V, Manjunath K. Preparation, characterization and in vitro release kinetics of clozapine solid lipid nanoparticles. J Control Release. 2004;95:627-38.

46. Torchilin PV. Structure and design of polymeric surfactantbased drug delivery systems. J Control Release. 2011;73:13772.

47. Bae YH, Yin HQ. Stability issues of polymeric micelles. J Control Release. 2008;131:2-4. 